\title{
On the homology theory of fiber spaces
}

\author{
T.V. Kadeishvili
}

\begin{abstract}
This paper was published (in Russian) in Uspekhi Mat. Nauk 35:3 (1980), 183-188. The English translation was published in Russian Math. Surveys, 35:3 (1980), 231-238.
\end{abstract}

In this paper the homology theory of fibre spaces is studied by introducing additional algebraic structure in homology and cohomology.

All modules are assumed to be over an arbitrary associative ring $\Lambda$ with unit; by a differential algebra, coalgebra, module, or comodule we mean these objects graded by non-negative integers; $\hat{a}$ denotes $(-1)^{\text {dega }}$.

The category $A(\infty)$. An $A(\infty)$-algebra in the sense of Stasheff 1$]$ is defined to be a graded $\Lambda$-module $M$, endowed with a set of operations $\left\{m_{i}: \otimes^{i} M \rightarrow\right.$ $M, i=1,2, \ldots\}$ satisfying the conditions $m_{i}\left(\left(\otimes^{i} M\right) q\right) \subset M_{q+i-2}$ and

$\sum_{k=0}^{i-1} \sum_{j=1}^{i-k}(-1)^{k} m_{i-j+1}\left(\hat{a}_{1} \otimes \ldots \otimes \hat{a}_{k} \otimes m_{j}\left(a_{k+1} \otimes \ldots \otimes a_{k+j}\right) \otimes a_{k+j+1} \otimes \ldots \otimes a_{i}\right)=0$

for any $a_{i} \in M$ and $i \geq 1$. A morphism of $A(\infty)$-algebras $\left(M,\left\{m_{i}\right\}\right) \rightarrow$ $\left(M^{\prime},\left\{m_{i}^{\prime}\right\}\right)$ is a set of homomorphisms $\left\{f_{i}: \otimes^{i} M \rightarrow M^{\prime}, i=1,2, \ldots\right\}$ satisfying the conditions $f_{i}\left(\left(\otimes^{i} M\right) q\right) \subset M_{q+i-1}^{\prime}$ and

$$
\begin{aligned}
& \sum_{k=0}^{i-1} \sum_{j=1}^{i-k}(-1)^{k} f_{i-j+1}\left(\hat{a}_{1} \otimes \ldots \otimes \hat{a}_{k} \otimes m_{j}\left(a_{k+1} \otimes \ldots \otimes a_{k+j}\right) \otimes \ldots \otimes a_{i}\right)= \\
& \sum_{t=1}^{i} \sum_{S(t, i)} m_{t}^{\prime}\left(f_{k_{1}}\left(a_{1} \otimes \ldots \otimes a_{k_{1}}\right) \otimes \ldots \otimes f_{k_{t}}\left(a_{i-k_{t}+1} \otimes \ldots \otimes a_{i}\right)\right)
\end{aligned}
$$

where $S(t, i)=\left\{k_{1}, \ldots, k_{t} \in N, \sum k_{p}=i\right\}$. The $A(\infty)$-algebras together with these morphisms form a category, which we denote by $A(\infty)$.

The specification on $M$ of an $A(\infty)$-algebra structure $\left(M,\left\{m_{i}\right\}\right)$ is equivalent to the specification on the tensor coalgebra $T^{c}(M)=\Lambda+M+M \otimes M+\ldots$ with the grading $\operatorname{dim}\left(a_{1} \otimes \ldots \otimes a_{n}\right)=\sum \operatorname{dima}_{i}+n$ and comultiplication

$$
\Delta\left(a_{1} \otimes \ldots \otimes a_{n}\right)=\sum_{i=0}^{n}\left(a_{1} \otimes \ldots \otimes a_{i}\right) \otimes\left(a_{i+1} \otimes \ldots \otimes a_{n}\right)
$$


of a differential $d_{m}: T^{c}(M) \rightarrow T^{c}(M)$ that turns $T^{c}(M)$ into a differential coalgebra; this set $\left\{m_{i}\right\}$ determines the differential $d_{m}$ by

$d_{m}\left(a_{1} \otimes \ldots \otimes a_{n}\right)=\sum_{k=0}^{n-1} \sum_{j=1}^{n-k}(-1)^{k} \hat{a}_{1} \otimes \ldots \otimes \hat{a}_{k} \otimes m_{j}\left(a_{k+1} \otimes \ldots \otimes a_{k+j}\right) \otimes \ldots \otimes a_{n}$

and the differential coalgebra $\left(T^{c}(M), d_{m}\right)$ is called the $\tilde{B}$-construction of the $A(\infty)$-algera $\left(M,\left\{m_{i}\right\}\right)$ (Stasheff [1] ) and is denoted by $\tilde{B}\left(M,\left\{m_{i}\right\}\right)$. The specification of an $A(\infty)$-algebra morphism $\left\{f_{i}\right\}:\left(M,\left\{m_{i}\right\}\right) \rightarrow\left(M^{\prime},\left\{m_{i}^{\prime}\right\}\right)$ is equivalent to that of a differential coalgebra mapping $f: \tilde{B}\left(M,\left\{m_{i}\right\}\right) \rightarrow \tilde{B}\left(M^{\prime},\left\{m_{i}^{\prime}\right\}\right)$; the morphism $\left\{f_{i}\right\}$ determines the mapping $f$ by

$$
f\left(a_{1} \otimes \ldots \otimes a_{n}\right)=\sum_{t=1}^{n} \sum_{S(t, n)} f_{k_{1}}\left(a_{1} \otimes \ldots \otimes a_{k_{1}}\right) \otimes \ldots \otimes f_{k_{t}}\left(a_{n-k_{t}+1} \otimes \ldots \otimes a_{i}\right) .
$$

Thus the category $A(\infty)$ can be identified with a full subcategory of the category of differential coalgebras.

An arbitrary object in $A(\infty)$ of the form $\left(M,\left\{m_{1}, m_{2}, 0,0, \ldots\right\}\right)$ is identified with the differential algebra $(M, \partial, \cdot)$ where $\partial=m_{1}$ and $a_{1} \cdot a_{2}=-m_{2}\left(\tilde{a}_{1} \otimes a_{2}\right)$. For such an object the $\tilde{B}$-construction coincides with the usual $B$-construction, any morphism of such objects of the form $\left\{f_{1}, 0,0, \ldots\right\}$ is identified with the differential algebra mapping $f_{1}:(M, \partial, \cdot) \rightarrow(M, \partial, \cdot)$. Thus the category of differential algebras is a subcategory of $A(\infty)$, while the category $D A S H$ (see [2]) is the full subcategory of $A(\infty)$ generated by differential algebras, and the functor $\tilde{B}$ is an extension of $B$ from this subcategory to $A(\infty)$.

Theorem 1 For any differential algebra $C$ with free $H_{i}(C), i \geq 0$ it is possible to introduce on $H(C)$ an $A(\infty)$-algebra structure

$$
\left(H(C),\left\{X_{i}\right\}\right), X_{i}: \otimes^{i} H(C) \rightarrow H(C), i=1,2,3, \ldots
$$

such that $X_{1}=0, X_{2}\left(a_{1} \otimes a_{2}\right)=-\tilde{a}_{1} \cdot a_{2}$ and there exists an $A(\infty)$-morphism

$$
\left\{f_{i}\right\}:\left(H(C),\left\{X_{i}\right\}\right) \rightarrow\left(C,\left\{m_{1}, m_{2}, 0,0, \ldots\right\}\right)
$$

for which $f_{1}: H(C) \rightarrow C$ induces an identical isomorphis in homology.

Proof. We need to construct two sets of homomorphisms

$$
\left.\left\{X_{i}: \otimes^{i} H(C) \rightarrow H(C), i=1,2,3, \ldots\right\},\left\{f_{i}: \otimes^{i} H(C) \rightarrow C\right), i=1,2,3, \ldots\right\},
$$

satisfying the conditions in the definition of the category $A(\infty)$ :

$$
\sum_{k=0}^{i-1} \sum_{j=1}^{i-k}(-1)^{k} X_{i-j+1}\left(\hat{a}_{1} \otimes \ldots \otimes \hat{a}_{k} \otimes X_{j}\left(a_{k+1} \otimes \ldots \otimes a_{k+j}\right) \otimes a_{k+j+1} \otimes \ldots \otimes a_{i}\right)=0,
$$




$$
\begin{aligned}
& \sum_{k=0}^{i-1} \sum_{j=1}^{i-k}(-1)^{k} f_{i-j+1}\left(\hat{a}_{1} \otimes \ldots \otimes \hat{a}_{k} \otimes X_{j}\left(a_{k+1} \otimes \ldots \otimes a_{k+j}\right) \otimes \ldots \otimes a_{i}\right)= \\
& m_{1} f_{i}\left(a_{1} \otimes \ldots \otimes a_{i}\right)+ \\
& \sum_{s=1}^{i-1} m_{2}\left(f_{s}\left(a_{1} \otimes \ldots \otimes a_{s}\right) \otimes f_{i-s}\left(a_{s+1} \otimes \ldots \otimes a_{i}\right)\right)
\end{aligned}
$$

for arbitrary $a_{k} \in H(C)$ and $i \geq 1$. Fir $i=1$ we take $X_{1}=0$, and, using the fact that $H_{i}(C)$ is free, we define $f_{1}: H(C) \rightarrow C$ to be a cycle-choosing homomorphis; the conditions (1I) and (2), as well as the initial condition on $f_{1}$ are thereby satisfied. Suppose now that $X_{i}$ and $f_{i}$ have been constructed for $i<n$ in such a way that the conditions (1) and (2) hold. Let

$$
\begin{aligned}
& U_{n}\left(a_{1} \otimes \ldots \otimes a_{n}\right)=\sum_{s=1}^{n-1} m_{2}\left(f_{s}\left(a_{1} \otimes \ldots \otimes a_{s}\right) \otimes f_{n-s}\left(a_{s+1} \otimes \ldots \otimes a_{n}\right)\right)+ \\
& \sum_{k=0}^{n-2} \sum_{j=2}^{n-1}(-1)^{k+1} f_{n-j+1}\left(\hat{a}_{1} \otimes \ldots \otimes \hat{a}_{k} \otimes X_{j}\left(a_{k+1} \otimes \ldots \otimes a_{k+j}\right) \otimes \ldots \otimes a_{n}\right)
\end{aligned}
$$

(here the $X_{i}$ and $f_{i}$ already defined are involved). Then the condition (2) takes the form

$$
m_{1} f_{n}\left(a_{1} \otimes \ldots \otimes a_{n}\right)=\left(f_{1} X_{n}-U_{n}\right)\left(a_{1} \otimes \ldots \otimes a_{n}\right) .
$$

Direct calculations show that $\partial U_{n}=0$, that is, $U_{n}\left(a_{1} \otimes \ldots \otimes a_{n}\right)$ is a cycle in $C$ for arbitrary $a_{i} \in H(C)$, and we define $X_{n}\left(a_{1} \otimes \ldots \otimes a_{n}\right)$ to be the class of this cycle, that is, $X_{n}=\left\{U_{n}\right\}$. Since $f_{1}$ is a cycle-choosing homomorphism, the difference $f_{1} X_{n}-U_{n}$ is homological to zero. Assuming that $a_{i} \in H(C)$ are free generators we define $f_{n}\left(a_{1} \otimes \ldots \otimes a_{n}\right)$ as an element of $C$ boundaring this difference and extend by linearity. For the $X_{n}$ and $f_{n}$ thus defined the condition (3) is automatically satisfied. The remanning condition (11) can be proved by a straightforward check.

We remark that the theorem is true also when an arbitrary $A(\infty)$-algebra is taken instead of $C$, and $H(M)$ is understood to be the homology of $M$ with respect to the differential $m_{1}$.

The $A(\infty)$-algebra $\left(H(C),\left\{X_{i}\right\}\right)$ we call the homology $A(\infty)$-algebra of the differential algebra $C$. As is clear from the proof, this structure is not uniquely determined on $H(C)$ (there is an arbitrariness in the choice of the $f_{i}$ ). We show later that the structure of the homology $A(\infty)$-algebra on $H(C)$ is unique up to isomorphism in $A(\infty)$.

We mention that if $a_{1} \cdot a_{2}=a_{2} \cdot a_{3}=0$ for $a_{1}, a_{2}, a_{3} \in H(C)$, then $X_{3}\left(a_{1} \otimes\right.$ $\left.a_{2} \otimes a_{3}\right)$ is an element of the Massey product $\left\langle a_{1}, a_{2}, a_{3}\right\rangle$, and this fact provides us with examples in which the operation $X_{3}$ is non-trivial. The next result follows from the Theorem [1]

Corollary 1 The mapping of differential coalgebras

$$
f: \tilde{B}\left(H(C),\left\{X_{i}\right\}\right) \rightarrow B(C)
$$

induces an isomorphism in homology.

The category $M(\infty)$. An $A(\infty)$-module over an $A(\infty)$-algebra $\left(M,\left\{m_{i}\right\}\right)$ we define to be a graded $\Lambda$-module $P$, endowed with a set of operations $\left\{p_{i}\right.$ : 
$\left.\left(\otimes^{i-1} M\right) \otimes P \rightarrow P, \quad i=1,2,3, \ldots\right\}$ satisfying the conditions $p_{i}\left(\left(\left(\otimes^{i-1} M\right) \otimes\right.\right.$ $\left.P)_{q}\right) \subset M_{q+i-2}$ and

$$
\begin{aligned}
& \sum_{k=0}^{i-2} \sum_{j=1}^{i-k-1}(-1)^{k} p_{i-j+1}\left(\hat{a}_{1} \otimes \ldots \otimes \hat{a}_{k} \otimes m_{j}\left(a_{k+1} \otimes \ldots \otimes a_{k+j}\right) \otimes \ldots \otimes\right. \\
& \left.a_{i-1} \otimes b\right)+\sum_{k=0}^{i-1}(-1)^{k} p_{k+1}\left(\hat{a}_{1} \otimes \ldots \otimes \hat{a}_{k} \otimes p_{i-k}\left(a_{k+1} \otimes \ldots \otimes a_{i-1} \otimes b\right)\right)=0 .
\end{aligned}
$$

The specification on $P$ of an $A(\infty)$-module structure over $\left(M,\left\{m_{i}\right\}\right)$ is equivalent to the specification on $\tilde{B}\left(M,\left\{m_{i}\right\}\right) \otimes P$ of a differential that turns it into a differential comodule over $\tilde{B}\left(M,\left\{m_{i}\right\}\right)$. The objects of the category $M(\infty)$ are defined to be the pairs $\left(\left(M,\left\{m_{i}\right\}\right),\left(P,\left\{p_{i}\right\}\right)\right)$, where $\left(M,\left\{m_{i}\right\}\right)$ is an $A(\infty)$ algebra, and $\left(P,\left\{p_{i}\right\}\right)$ is an $A(\infty)$-module over it. A morphism is defined to be a pair of sets of homomorphisms $\left\{f_{i}\right\},\left\{g_{i}\right\}$ where $\left\{f_{i}\right\}:\left(M,\left\{m_{i}\right\}\right) \rightarrow\left(M^{\prime},\left\{m_{i}^{\prime}\right\}\right)$ is a morphism of $A(\infty)$-algebras and

$$
\left\{g_{i}:\left(\otimes^{i-1} M\right) \otimes P \rightarrow P^{\prime}, i=1,2,3, \ldots\right\}
$$

is a set satisfying the conditions $g_{i}\left(\left(\left(\otimes^{i-1} M\right) \otimes P\right)_{q}\right) \subset P_{q+i-1}^{\prime}$ and

$$
\begin{aligned}
& \sum_{k=0}^{i-2} \sum_{j=1}^{i-k-1}(-1)^{k} \\
& g_{i-j+1}\left(\hat{a}_{1} \otimes \ldots \otimes \hat{a}_{k} \otimes m_{j}\left(a_{k+1} \otimes \ldots \otimes a_{k+j}\right) \otimes \ldots \otimes a_{i-1} \otimes b\right)= \\
& \sum_{t=1}^{i} \sum_{S(t, i)} p_{t}\left(f_{k_{1}}\left(a_{1} \otimes \ldots \otimes a_{k_{1}}\right) \otimes f_{k_{2}}\left(a_{k_{1}+1} \otimes \ldots \otimes a_{k_{1}+k_{2}}\right) \otimes \ldots \otimes\right. \\
& \left.f_{k_{t-1}}\left(a_{k_{1}+\ldots+k_{t-2}+1} \otimes \ldots \otimes a_{k_{1}+\ldots+k_{t-1}}\right) \otimes g_{k_{t}}\left(a_{i-k_{t}+1} \otimes \ldots \otimes a_{i-1} \otimes b\right)\right)
\end{aligned}
$$

These conditions ensure that the mapping

$$
g: \tilde{B}\left(M,\left\{m_{i}\right\}\right) \otimes P \rightarrow \tilde{B}\left(M^{\prime},\left\{m_{i}^{\prime}\right\}\right) \otimes P^{\prime}
$$

given by

$$
\begin{aligned}
& g\left(a_{1} \otimes \ldots \otimes a_{i-1} \otimes b\right)=\sum_{t=1}^{i} \sum_{S(t, i)} f_{k_{1}}\left(a_{1} \otimes \ldots \otimes a_{k_{1}}\right) \otimes \ldots \otimes \\
& f_{k_{t-1}}\left(a_{k_{1}+\ldots+k_{t-2}+1} \otimes \ldots \otimes a_{k_{1}+\ldots+k_{t-1}}\right) \otimes g_{k_{t}}\left(a_{k_{1}+\ldots+k_{t-1}+1} \otimes \ldots \otimes a_{i-1} \otimes b\right)
\end{aligned}
$$

is a differential comodule mapping compatible with $f$. With the obvious morphisms the category of pairs $(C, D)$, where $C$ is a differential algebra and $D$ is a differential module over it, forms a subcategory of $M(\infty)$.

Theorem 2 If $C$ is a differential algebra and $D$ is a differential module over it such that $H_{i}(C)$ and $H_{i}(D)$ are free, then on $H(D)$ it is possible to introduce the structure of an $A(\infty)$-module $\left(H(D),\left\{Y_{i}\right\}\right), Y_{i}:\left(\otimes^{i-1} H(C)\right) \otimes$ $H(D) \rightarrow H(D), p=1,2,3, \ldots$ over the homology $A(\infty)$-algebra $\left(H(C),\left\{X_{i}\right\}\right)$ such that $Y_{1}=0, Y_{2}(a \otimes b)=-\tilde{a} \cdot b$ and there exists a morphism $\left(\left\{f_{i}\right\},\left\{g_{i}\right\}\right)$ : $\left(\left(H(C),\left\{X_{i}\right\}\right),\left(H(D),\left\{Y_{i}\right\}\right)\right) \rightarrow(C, D)$ of $M(\infty)$ for which $f_{1}: H(C) \rightarrow C$ and $g_{1}: H(D) \rightarrow D$ induce identical isomorphisms in homology.

Proof. The sets $\left\{g_{i}\right\}$ and $\left\{Y_{i}\right\}$ are constructed by induction on $i$ just as in the proof of Theorem [1] Using the fact that $H_{i}(D)$ is free, we define $g_{1}$ to be a 
cycle-choosing homomorphism, while $Y_{1}=0$, and the conditions of the category $M(\infty)$ are satisfied for $i=1$. Let

$$
\begin{aligned}
& \left.V_{n}\left(a_{1} \otimes \ldots \otimes a_{n-1} \otimes b\right)\right)= \\
& \sum_{s=1}^{n-1} p_{2}\left(f_{s}\left(a_{1} \otimes \ldots \otimes a_{s}\right) \otimes g_{n-s}\left(a_{s+1} \otimes \ldots \otimes a_{n-1} \otimes b\right)\right)+ \\
& \sum_{k=0}^{n-3} \sum_{j=2}^{n-1}(-1)^{k+1} g_{i-j+1}\left(\hat{a}_{1} \otimes \ldots \otimes \hat{a}_{k} \otimes X_{j}\left(a_{k+1} \otimes \ldots \otimes a_{k+j}\right) \otimes \ldots \otimes\right. \\
& \left.a_{n-1} \otimes b\right)+\sum_{k=1}^{n-2}(-1)^{k} g_{k+1}\left(\hat{a}_{1} \otimes \ldots \otimes \hat{a}_{k} \otimes Y_{n-k}\left(a_{k+1} \otimes \ldots \otimes a_{n-1} \otimes b\right)\right),
\end{aligned}
$$

then $\partial V_{n}=0$, therefore, we define $Y_{n}=\left\{V_{n}\right\}$. Since $g_{1}$ is a cycle-choosing homomorphism, $g_{1} Y_{n}-V_{n}$ is a cycle in $D$ homologous to zero. Using the fact that $H_{i}(D)$ is free, we define $g_{n}:\left(\otimes^{n-1}\right) \otimes H(D) \rightarrow H(D)$ to be a homomorphism for which $\partial g_{n}=g_{1} V_{n}-V_{n}$. The conditions of the category $M(\infty)$ are satisfied for the $Y_{n}$ and $g_{n}$ thus defined.

Twisted tensor products. The twisted tensor products of Brown 3 can be generalized from the case of differential algebras and modules to the case of $A(\infty)$-algebras and $A(\infty)$-modules: for an arbitrary differential coalgebra $(K, d)$ and an $A(\infty)$-algebra $\left(M,\left\{m_{i}\right\}\right)$ a $\sim$-twisting cochain we define to be a homomorphism $\phi: K \rightarrow M$ of degree -1 that satisfies the condition

$$
\phi d=\sum_{i=1}^{\infty} m_{i}(\phi \otimes \phi \otimes \ldots \otimes \phi) \Delta^{i},
$$

where $\Delta^{i}: K \rightarrow K \otimes K$ is the homomorphism defined by $\Delta^{1}=i d_{K}, \Delta^{2}=$ $\Delta: K \rightarrow K \otimes K, \Delta^{i}=\left(i d_{K} \otimes \Delta^{i-1}\right) \Delta$. The specification of a $\sim$-twisting cochain $\phi: K \rightarrow M$ is equivalent to that of a mapping of differential coalgebra $f_{\phi}:(K, d) \rightarrow \tilde{B}\left(M,\left\{m_{i}\right\}\right)$. For any $(K, d)$ and $\left(\left(M,\left\{m_{i}\right\}\right),\left(P,\left\{p_{i}\right\}\right)\right) \in M(\infty)$ any $\sim$-twisting cochain $\phi: K \rightarrow M$ on the tensor product $K \otimes P$ determines by

$$
\partial_{\phi}=d \otimes i d_{P}+\sum_{i=1}^{\infty}\left(\hat{i d} \otimes p_{i}\right)\left(i d_{K} \otimes \phi \otimes \ldots \otimes \phi \otimes i d_{P}\right)\left(\Delta^{i} \otimes i d_{P}\right)
$$

a differential, turning $\left(K \otimes P, \partial_{\phi}\right)$ into a differential comodule over $(K, d)$; this differential comodule is called the $\sim$-twisted tensor product $K \otimes_{\phi} P$. If $M$ is an $A(\infty)$-algebra of the form $\left(M,\left\{m_{1}, m_{2}, 0,0, \ldots\right\}\right)$, and $P$ is an $A(\infty)$-module of the form $\left(P,\left\{p_{1}, p_{2}, 0,0, \ldots\right\}\right)$, then $\phi$ is the usual twisting cochain, and $K \otimes_{\phi} P$ coincides with the usual twisted tensor product $K \otimes_{\phi} P$.

We need the concept of equivalence of twisting cochains (see [4], [5], [6]). We say that $\phi, \psi: K \rightarrow C$ are equivalent if there is a homomorphism $c: K \rightarrow C$ of degree 0 for which $c_{0}=c \mid C_{0}=0$ and $\psi=(1+c) \star \phi$ where

$$
(1+c) \star \phi=(1+\hat{c}) \cdot \phi \cdot(1+c)^{-1}-(c d+\partial c) \cdot(1+c)^{-1} ;
$$

$\phi \sim \psi$ if and only if $f_{\phi}, f_{\psi}: K \rightarrow B$ are homotopic in the sense of [2] (coderivation homotopy): $f_{\phi}-f_{\psi}=\partial D+D \partial$ with $\left(D \otimes f_{\psi}+\hat{f}_{\phi} \otimes D\right) \Delta=\Delta D$.

Theorem 3 If $(K, d)$ is a differential coalgebra with free $K_{i}$, and $\phi: K \rightarrow C$ is an arbitrary twisting cochain, then there exists a $\sim$-twisting cochain $\phi^{*}: K \rightarrow$ $H(C)$ such that $\phi$ and $f^{*} \phi^{*}=\sum_{i=1}^{\infty} f_{i}(\phi * \otimes \ldots \otimes \phi *) \Delta^{i}$ are equivalent. 
Proof. To construct $\phi *$ we prove the following inductive assertion: for any $i>0$ there exists a twisting cochain $\phi^{(i)}: K \rightarrow C$ and a homomorphisms $\phi_{i}^{*}: K_{i} \rightarrow H_{i-1}(C)$ and $c_{i}: K_{i} \rightarrow C_{i}$ such that

$$
\begin{aligned}
& \text { (a) } \phi_{i}^{*} d=\sum_{t=2}^{i} \sum_{S(t, i)} X_{t}\left(\phi_{k_{1}}^{*} \otimes \ldots \otimes \phi_{k_{t}}^{*}\right) \Delta^{t} ; \\
& \text { (b) } \phi^{(i)}=\left(1+c_{i}\right) \star \phi^{(i-1)} \\
& \text { (c) } \phi_{i}^{(i)}=\sum_{t=1}^{i} \sum_{S(t, i)} f_{t}\left(\phi_{k_{1}}^{*} \otimes \ldots \otimes \phi_{k_{t}}^{*}\right) \Delta^{t} .
\end{aligned}
$$

For $i=1$ we take $\phi_{1}^{*}=\left\{\phi_{1}\right\}$. Since the difference $\left(\phi_{1}-f_{1} \phi_{1}^{*}\right)(k)$ is homologous to zero for each $k \in K_{1}$ and $K_{1}$ is free, we obtain a homomorphism $c_{1}: K_{1} \rightarrow C_{1}$ for which $-\partial c_{1}=\phi_{1}-f_{1} \phi_{1}^{*}$. We define $\phi^{(1)}=\left(1+c_{1}\right) \star \phi$, so $\phi_{1}^{(1)}=\phi_{1}+\partial c_{1}=f_{1} \phi_{1}^{*}$. Suppose now that $\phi^{(i)}, \phi_{i}^{*}$, and $c_{i}$ have already been constructed in such a way that $(a),(b)$, and $(c)$ hold for $i<n$. Let

$$
W_{n}=\phi_{n}^{(n-1)}-\sum_{t=2}^{n} \sum_{S(t, n)} f_{t}\left(\phi_{k_{1}}^{*} \otimes \ldots \otimes \phi_{k_{t}}^{*}\right) \Delta^{t} ;
$$

A direct check shows that $\partial W_{n}=0$; we define $\phi_{n}^{*}=\left\{W_{n}\right\}$. Since the difference $W_{n}-f_{1} \phi_{n}^{*}$ is homological to zero and $K_{n}$ is free, we can construct a $c_{n}: K_{n} \rightarrow$ $C_{n}$ such that $-\partial c_{n}=W_{n}-f_{1} \phi_{n}^{*}$; let $\phi^{(n)}=\left(1+c_{n}\right) \star \phi^{(n-1)}$. Then

$$
\phi_{n}^{(n)}=\phi_{n}^{(n-1)}+\partial c_{n}=f_{1} \phi_{n}^{*}-W_{n}=\sum_{t=1}^{n} \sum_{S(t, n)} f_{t}\left(\phi_{k_{1}}^{*} \otimes \ldots \otimes \phi_{k_{t}}^{*}\right) \Delta^{t},
$$

consequently, $(b)$ and $(c)$ hold for $\phi^{(n)}, \phi_{n}^{*}$, and $c_{n}$, and the validity of $(a)$ can be checked directly. From $(a)$ we see that $\phi^{*}=\sum_{i} \phi_{i}^{*}$ is a $\sim$-twisting cochain, and from $(b)$ and $(c)$ we deduce that $f^{*} \phi^{*}=\phi^{\infty}$, where $\phi^{\infty}=\Pi_{i}\left(1+c_{i}\right) \star \phi \sim \phi$.

It follows from Theorem 3 that for any differential coalgebra mapping $g$ : $K \rightarrow B$ there exists a $G^{*}: K \rightarrow \tilde{B}\left(H(C),\left\{X_{i}\right\}\right)$ for which $g$ and $f g^{*}$ are homotopic in the sense of [2]. This assertion implies uniqueness mentioned above for the structure of homology $A(\infty)$-algebra: if $\left(H(C),\left\{X_{i}\right\}\right)$ and $\left(H(C),\left\{X_{i}\right\}\right)$ are two structures of homology $A(\infty)$-algebra on $H(C)$, then by taking $K=$ $\tilde{B}\left(H(C),\left\{X_{i}\right\}\right)$ and $g=f: \tilde{B}\left(H(C),\left\{X_{i}\right\}\right) \rightarrow B(C)$, we obtain a

$$
g^{*}: \tilde{B}\left(H(C),\left\{X_{i}\right\}\right) \rightarrow \tilde{B}\left(H(C),\left\{X_{i}\right\}\right)
$$

for which $f g^{*} \sim g$. Then the first component of the $A(\infty)$ algebra morphism $\left\{g_{i}^{*}\right\}:\left(H(C),\left\{X_{i}\right\}\right) \rightarrow\left(H(C),\left\{X_{i}\right\}\right)$ induced by $g^{*}$ is $g_{1}^{*}=i d_{H(C)}$, and this implies that $\left\{g_{i}^{*}\right\}$ is an isomorphism in $A(\infty)$.

The next result follows from Theorems 10 and 3

Corollary $2 K \otimes_{\phi} D$ and $K \otimes_{\phi^{*}} H(D)$ have isomorphic homology under the conditions of Theorems 1 , 目 and 3 .

The results obtained have the following applications.

The first proposition is obtained from Corollary 1 by taking $C=\bar{C}_{*}(G)$, where $G$ is a connected topological group such that the $H_{i}(G)$ are free, bearing in mind that $H(B(C))=H_{*}\left(B_{G}\right)$. 
Proposition 1 The homology of the $\tilde{B}$-construction $\tilde{B}\left(\bar{H}_{*}(G),\left\{X_{i}\right\}\right)$ are isomorphic to that of classifying space $B_{G}$.

The next proposition is obtained from Corollary 1 by taking $C=C^{*}\left(B, b_{0}\right)$, where $B$ is a simply connected space with free groups $H^{i}\left(B, b_{0}\right)$, and bearing in mind that $H(B(C))=H^{*}(\Omega C)$.

Proposition 2 The homology of the $\tilde{B}$-construction $\tilde{B}\left(\bar{H}^{*}\left(B, b_{0}\right),\left\{X_{i}\right\}\right)$ are isomorphic to the cohomology of the loop space $\Omega B$.

Let $\xi=(X, p, B, G)$ be a principal $G$-fibration with paracompact base and connected $G$, let $F$ be a $G$-space, and $\xi[F]=(E, p, B, F, G)$ the associated fiber bundle, with the $H_{i}(G)$ and $H\left({ }_{i}(F)\right.$ free. The final proposition is obtained from Corollary 2 ty taking $C=C_{*}(G), D=C_{*}(F)$, and $\phi$ a twisting cochain of the fibration $\xi(\underline{3})$.

Proposition 3 The homology of the $\sim$-twisted tensor product $C_{*}(B) \otimes_{\phi^{*}} H_{*}(F)$ is isomorphic to that of $E$.

This proposition generalize a result of Shih [7]: if $G$ is $(n-1)$-connected, then the components $\phi_{i}^{*} \in C^{i}\left(B, H_{i-1}(G)\right)$ vanish for $0<i<n+1$, therefore, the differentials $d^{i}$ of the spectral sequence of $\xi[F]$ are trivial for $1<i<n+1$, and the components are cocycles for $n<i<2 n+1$, consequently, $d^{j}$ can be expressed for $n<i<2 n+1$ in terms of certain characteristic classes of $\xi$ and the operation $Y_{2}: H_{*}(G) \otimes H_{*}(F) \rightarrow H_{*}(F)$; we remark that higher operations $Y_{i}$ are needed for computing $d^{j}, j>2 n$, in terms of $\phi^{*}$.

Theorems 10 and Proposition 3 were announced in 8 and Proposition 2 in 9 .

\section{References}

[1] J.D. Stasheff, Homotopy associativity of H-spaces, I, II, Trans. Amer. Math. Soc. 108 (1963), 27-313. MR 281623.

[2] H. Munkholm, The Eilenberg-More spectral sequence and strongly homotopy multiplicative maps, J. Pure and Appl. Algebra 5 (1974), 1-50. MR 503227.

[3] E. H. Brown, Twisted tensor products, I, Ann. Of Math. (2) 69 (1959), 223-246. MR 214423.

[4] N. A. Berikashvili, The differentials of a spectral sequence, Bull. Of Georg. Acad. Sci., 51 (1968), 9-14. MR 419258.

[5] N. A. Berikashvili, Homology theory of spaces, Bull. Of Georg. Acad. Sci., 86 (1977), 529-532. MR 5713949.

[6] V. A. Smirnov, The functor $D$ for twisted tensor products, Mat. Zametki 20 (1976), 465-472. MR 554172. 
[7] W. Shih, Homologie des espaces fibres, Inst. Hautes Etudes Sci. Publ. Math. 1962, no. 13, 88. MR 261893.

[8] T. Kadeishvili, On the differentials of spectral sequence of a fiber bundle, Bull. Of Georg. Acad. Sci., 82 (1976), 285-288. MR 556430.

[9] T. Kadeishvili, On the homology of classifying spaces, Proc. 7th All Union Topology Conference, Minsk 1977. 